\title{
Propos sur les paysages de catastrophe : Tchernobyl
}

\section{Henri-Pierre Jeudy}

\section{(2) OpenEdition}

1 Journals

\section{Édition électronique}

URL : http://journals.openedition.org/leportique/2133

DOI : $10.4000 /$ leportique.2133

ISSN : $1777-5280$

\section{Éditeur}

Association "Les Amis du Portique"

\section{Édition imprimée}

Date de publication : 10 avril 2009

ISSN : 1283-8594

\section{Référence électronique}

Henri-Pierre Jeudy, «Propos sur les paysages de catastrophe: Tchernobyl », Le Portique [En ligne], 22 I 2009, mis en ligne le 10 novembre 2010, consulté le 26 mars 2021. URL : http://

journals.openedition.org/leportique/2133; DOI : https://doi.org/10.4000/leportique.2133

Ce document a été généré automatiquement le 26 mars 2021.

Tous droits réservés 


\title{
Propos sur les paysages de catastrophe : Tchernobyl
}

\author{
Henri-Pierre Jeudy
}

1 «Faut-il réparer un paysage sinistré? Lui redonner un aspect "pictural" ? Une singularité nouvelle? Ou bien le laisser tel qu'il est. Il est intéressant de tenter de répondre à de telles questions en partant de la photographie. Quand on regarde les images d'un paysage dévasté par une exploitation industrielle massive et offrant l'aspect d'un aérolithe pelé et infertile, on éprouve des sentiments mêlés. Ce paysage est le résultat d'un travail photographique qui donne à un territoire ravagé une puissance suggestive dont on ne comprend pas très bien la nature mais qui produit sur nous un sentiment de rejet et de satisfaction mêlés» (Gilles Tiberghien). C'est la photographie qui semble a priori nous mettre en état de mieux appréhender « ce qui est en puissance " dans un territoire sinistré, comme si elle avait la capacité de nous révéler ce que nous ne pourrions pas voir «à l'œil nu». C'est elle qui restituerait à notre regard «l'état des choses », ce qui est, ce qui se dérobe à notre perception et qui pourtant fait la condition de ce que nous voyons. En somme, la photographie serait au plus près des sensations que nous éprouvons face à un paysage, qu'il soit sinistré ou non. Est-ce vraiment sûr?

2 Il y a, en ce qui fait paysage pour le regard, une densité incommensurable des choses qui se présentent dans le champ de la vision. Et cette impression de densité abolit la distinction entre ce qui vient de nous, des projections de notre "intérieur " et ce que nous voyons à l'extérieur. L'espace du dedans entre en coïncidence avec l'espace du dehors. Notre subjectivité se perd dans l'objectivité de l'état des choses que révèle le paysage. Quand quelqu'un arrive sur un territoire qu'il a connu depuis son enfance, il aime signaler qu'il reconnaît tous les détails du paysage. Cette imprégnation des lieux dans la mémoire visuelle donne à la répétition de la vision familière une joie qui outrepasse le seul plaisir de la reconnaissance. Les différents aspects du territoire ont été intériorisés pour devenir une sorte de paysage éternel qui pourrait être perçu les yeux fermés. Une relation d'intimité s'est établie entre le sujet et ce qu'il a toujours vu de telle manière que le paysage semble lui-même la conserver comme un bien précieux 
dont il serait le dépositaire. Curieusement, c'est à partir d'une pareille fusion que naît une sensation d'extériorité du paysage le plus familier, comme si, pour vivre l'impression de le retrouver, s'imposait une nécessité impérieuse de sortir de soi. Il ne s'agit pas d'une projection anthropomorphique, d'une personnalisation abusive du paysage devenu « mémoire vivante ». Advenant au regard habitué avec tous ses secrets connus ou méconnus, le paysage familier s'extériorise en ouvrant les voies de l'inconnu dans le mouvement même de la réitération.

3 L'attraction qu'exerce le paysage pour notre regard annihile la volonté, le vouloir voir. L'état physique et mental dans lequel nous nous trouvons quand nous regardons un paysage nous met dans une étrange confusion entre l'intériorité et l'extériorité. Il y a, dans la réciprocité entre ce qui fait objet pour notre regard et ce que nous sommes en train de voir, un mouvement d'intériorisation qui, venant de l'objet même, nous projette à l'extérieur de nous-mêmes, nous fait sortir de soi. Un paysage peut ainsi laisser advenir dans le regard sa propre intimité, ou du moins, ce que nous désignons comme tel, pour appréhender combien l'objet de notre vision, du fait de son extériorité, impulse des effets d'intériorisation. C'est dans le temps du regard que s'opère une perte de soi provoquée par l'extériorité du paysage et qu'émerge son intimité - la douceur du paysage. Dans son livre «l'expérience intérieure », Georges Bataille écrit : «Au moment où le jour décline, où le silence envahit un ciel de plus en plus pur, je me trouvais seul, assis dans une étroite véranda blanche, ne voyant rien d'où j'étais que le toit d'une maison, la frondaison d'un arbre et le ciel. Avant de me lever pour aller dormir, je sentis à quel point la douceur des choses m'avait pénétré. Je venais d'avoir le désir d'un mouvement d'esprit violent et, dans ce sens, j'aperçus que l'état de félicité où j'étais tombé ne différait pas entièrement des états "mystiques". Tout au moins, comme j'étais passé brusquement de l'inattention à la surprise, je ressentis cet état avec plus d'intensité qu'on ne fait d'habitude et comme si un autre et non moi l'éprouvait.» Ce mouvement d'extériorité par rapport à soi-même que provoque le paysage, qu'il soit inconnu ou familier, nous donne cette impression que ce n'est plus nous-mêmes qui regardons, que la douceur des choses que nous voyons nous pénètre en nous projetant comme si nous étions «autre ». Ce n'est pas cet état de fusion que laisse à penser la contemplation, c'est une séparation de soi qui s'opère dans l'imprégnation des choses sur le regard suspendu par le pur état des choses.

4 Et la puissance du paysage entre en nous sans la moindre effraction comme un mouvement qui va nous rendre habité par l'état des choses. Nous perdons alors l'habituelle acuité de perception qui nous permet de distinguer ce que nous sommes en train de regarder. La description elle-même n'a plus de sens, elle relève trop d'une volonté de distinction qui nous rappelle notre position de sujet regardant. Mais ce qui constitue le paysage comme objet demeure toujours présent. «L'esprit s'éveillant à la vie intérieure est pourtant en quête d'un objet. Il renonce à l'objet que l'action propose pour un objet de nature différente, mais ne peut se passer d'objet: son existence ne peut se fermer sur elle-même. » L'objectalité du paysage reste la condition d'une extase de l'intériorité projetée hors d'elle-même.

5 Si je prends pour exemple le site de Tchernobyl, je peux, bien entendu, m'attacher à la perception du "sarcophage». Ce réacteur enveloppé par une chape de béton se présente dans le champ de ma vision comme le symbole majeur de la catastrophe passée. Il cristallise à lui tout seul toute la perception des lieux en imposant au regard le souvenir impérissable du désastre, de son histoire inoubliable. Mais en parcourant 
ces mêmes lieux, je vois aussi que la nature est devenue luxuriante, que des traces des villages disparus sont enfouies dans les hautes herbes, je sens que le site dans son ensemble est plongé dans un étrange silence... Toutes ces sensations adviennent simultanément comme si j'étais happé par une atmosphère dont chaque détail ne fait que conforter mon impression de densité du paysage.

Dans quelle mesure cette intimité du paysage sinistré peut-elle être saisie? Les photographes qui viennent prendre des milliers d'images pour rendre publiques les ruines de la catastrophe sont trop soucieux de capter une vérité du réel, de donner des preuves de ce qui est, de ce qui menace encore. Obsédés par l'idée de toujours dévoiler ce qui est caché, ce qui se dérobe au regard, ils risquent de ne jamais faire apparaître cette intimité du paysage. À quelques kilomètres du site de Tchernobyl, la ville de Prypiat qui a été abandonnée quelques jours après la catastrophe, présente des ruines qui font l'objet de nombreuses incursions de photographes artistes parce qu'elles paraissent encore très « vivantes » comme si les habitants venaient de quitter les lieux. On pénètre pour ainsi dire dans l'intimité de la ville déserte. Maryvonne Arnaud parcourt ce genre de territoire qu'elle désigne comme un " lieu à l'identité extrême ", elle photographie surtout le sol à ses pieds en ne cherchant aucune perspective, en vivant son rapport au site sinistré comme dans un « corps à corps ». Elle n'organise pas le champ de perception par souci de donner des informations, ou de montrer ce qui s'est passé, elle condense dans l'image, la relation de ses pas, de sa marche, à la densité du territoire. Densité qui finit par être aussi celle des informations. Ses fragments photographiques sont reproduits à leur échelle réelle, juxtaposés côte à côte pour reconstituer le parcellaire du sol d'origine. Ainsi, notre regard est-il convié à partager une intimité de ce paysage encore lourd de menaces à partir de sa banalité même.

7 "L'identité extrême » n'est plus nominative, elle appelle la reconnaissance publique de ce qui contient le non identifiable. Ce que l'art révèle des effets de la catastrophe, c'est le paradoxe de l'identité méconnaissable ou c'est la manière dont l'identité bascule dans l'irreprésentable non plus comme signe de la reconnaissance mais comme interrogation existentielle sans réponse possible. Les photographies de Maryvonne Arnaud font apparaître, par la façon dont la densité du territoire sinistré s'appréhende pour ainsi dire "en à plat ", l'invisible comme anéantissement de ce qui est mis en perspective. L'irreprésentable ne peut se penser que dans l'anéantissement de la perspective, tel l'effondrement de la profondeur de champ que nous prêtons aux constructions de nos représentations. Dans les désastres, il y a toujours une difficulté terrible à identifier les cadavres, à leur donner un nom, les visages étant si mutilés qu'ils sont rendus méconnaissables. Ce sont des visages sans relief. Et l'horreur qu'ils provoquent semble appeler l'identification comme un moyen de conjurer la néantisation des corps. Le visage non identifiable se fait lui-même territoire.

8 À Tchernobyl, l'intimité du paysage tient aussi à la présence des rares personnes qui demeurent encore sur le site, comme des survivants. Ces personnes-là sont porteuses d'un regard dans notre propre regard, même si nous ignorons la manière dont elles voient les choses en l'état. Ce regard qui se glisse dans notre regard est proche du regard de l'artiste qui, pour mettre en œuvre une connivence entre toutes ces différentes intimités, est appelé à utiliser pareil principe de l'emboîtement des regards, de leur réciprocité, dans la perspective d'une absence de finalité. Il y a pour ainsi dire une souveraineté singulière du paysage sinistré qui donne à celui-ci la figure possible de son intimité. 
9 Je préfère raconter la visite des lieux. L'autocar dans lequel nous avons pris place, vient de s'arrêter à l'entrée de la zone interdite. Pour entrer dans le territoire contaminé autour de Tchernobyl, il faut passer les barrières d'une douane comme si on pénétrait dans un autre pays dont il est difficile d'oublier qu'il est tenu à l'écart du monde. Ceux ou celles qui, parmi les passagers, disposent d'un compteur Geiger, décident de vérifier le degré de contamination. Rien d'alarmant. Chacun regarde les alentours, certains prennent des photographies. D'un côté ou de l'autre des clôtures, la nature demeure identique, son aspect luxuriant ne délivre aucun signe de la catastrophe passée. Dans la zone contaminée, à plusieurs reprises, l'autocar ralentit, se range sur le bord de la route, le guide invite ses passagers à descendre pour voir une pancarte indiquant la présence d'un village enfoui sous la terre. L'herbe haute, les arbustes, et même de grands arbres ont chassé toute trace d'une vie humaine. Les noms de ces villages sont inscrits sur des rectangles d'étoffe dans le couloir d'entrée du musée à Kiev. Une centaine environ. Et là, sur le territoire contaminé, ces mêmes noms perdus dans la végétation. Là encore, la nature sereine a l'air de se moquer des effets d'un désastre en étalant ses richesses comme si rien, absolument rien, ne s'était produit. L'autocar roule vers Tchernobyl sur une route toujours aussi droite. L'entrée dans la ville donne une étrange impression de désolation. Et cette couleur jaune, ce jaune qui lui rappelle l'époque où il a eu une hépatite. Il était très faible, il avait du mal à bouger les bras et les jambes, elle était restée auprès de lui, plus que jamais, son regard d'enfant lui rappelait leurs premiers baisers. Chacun observe ses bras, ils ne sont pourtant pas jaunes, chacun ressent des picotements sous sa peau. Il y a un café ouvert, on n'aperçoit personne à l'intérieur, les vitres sont si opaques que la lumière du jour ne semble pas y pénétrer. La végétation a poussé le long des artères avec une telle intensité que les branches d'arbre se cognent contre l'autocar. Les longues tuyauteries disposées en réseau à quelques centimètres du sol ne distribuent plus le gaz pour le chauffage public ou privé, elles ressemblent à des cordages qui délimiteraient le parcours d'un territoire abandonné en pleine nature. L'autocar s'arrête devant une maison qui fait office de tourisme où l'on vend des livres, mais pas encore de souvenirs.

Dans une salle, un rescapé explique, après avoir décrit son état déplorable, les moments de la catastrophe sans avoir l'air particulièrement passionné par sa propre narration. Il montre des tableaux statistiques, des photographies, des cartes... Chacun observe le visage de cet homme accablé par la répétition du même récit. Sa voix est neutre, elle fait écho au silence des lieux. Au cœur d'un territoire irradié, toutes les figurations des cancers deviennent possibles. L'horreur est irreprésentable. Depuis la fenêtre de la salle, on peut voir les rares humains qui se déplacent dans les rues, on les voit toujours de loin comme s'il était physiquement impossible de s'en approcher, ils ne ressemblent en rien à des morts-vivants, ils sont comme les gardiens égarés de ce qui continue à vivre sous nos yeux quand nous n'avons plus rien à voir.

11 L'autocar s'est ensuite arrêté près d'une sculpture érigée à la mémoire des liquidateurs. Ces derniers ont fabriqué le sarcophage en béton dont la fonction était d'arrêter au plus vite la propagation de la pollution mortelle dans l'atmosphère. Les soldats du contingent, dit-on, pouvaient à l'époque être libérés immédiatement de leurs obligations militaires s'ils acceptaient de passer deux minutes "en enfer». Le sarcophage ressemble à la carapace noire d'un gigantesque hanneton. Il se fissure, peut-être ce coléoptère monstrueux est-il en train de se soulever, une seconde enveloppe va le recouvrir afin d'éviter à nouveau le pire. L'horreur n'est pas le pire, car 
le pire on l'attend, on l'envisage, tandis que l'horreur, elle surgit. Le pire, c'est l'étalon des risques. En approchant d'un canal, on tente d'apercevoir un poisson difforme dans l'eau d'un canal. Il y a bien une grande tâche blanchâtre qui s'agite, celle-ci ne semble pourtant pas vouloir adopter la forme d'un poisson. Le monstre est là, peu visible, on soupçonne sa présence. Ce poisson est devenu très gros parce qu'il est trop nourri et que personne ne le pêche pour le manger.

12 L'autocar arrive dans une autre ville abandonnée, Pripyat. Les passagers descendent, inquiets du degré de contamination qu'ils sont en train de supporter. Le soleil inonde de sa lumière les ruines irradiées. On avance vers la place abandonnée, les dalles de béton qui recouvrent le sol sont fissurées, l'herbe pousse dans les fentes, on s'arrête devant le grand hôtel dont les vitres sont brisées. Le terrain d'une fête foraine, une roue géante avec ses coques rouillées, une autostrade avec ses auto-tamponneuses, des balançoires tordues, on aperçoit au loin un immeuble dont les pièces du rez-dechaussée sont couvertes de livres déchirés. Tout est resté en l'état pour tourner un film. La cité abandonnée depuis le jour de l'explosion. Vingt ans plus tard, elle n'a sans doute pas changé. Ce ne sont pas des ruines, ce ne seront peut-être jamais des ruines. Tout semble rester en l'état comme si le béton résistait à l'usure du temps. Les voix ellesmêmes ont quitté le silence, il n'y a plus d'écho. Les souvenirs irradiés. L'autocar repart. Il s'engage à nouveau sur la route droite jusqu'à Tchernobyl. Fantômes de la catastrophe, les rares personnes qui travaillent sur le site ne s'approchent pas des visiteurs.

13 Le secret d'un paysage, c'est la manière dont, pour le regard, celui-ci bascule dans l'irreprésentable. Les territoires sinistrés nous invitent à voir ce qui n'est pas en ce qui est, en ce qui demeure visible. Il ne s'agit pas des traces repérables qui permettent une lecture toujours possible de ce qui s'est passé, ou une mise en représentation d'une catastrophe ancienne. Le temps produit une dissolution de la représentation en imposant au regard la puissance de l'irreprésentable. Il est difficile de concevoir comment le paysage génère sa propre abstraction grâce à sa densité. C'est pourtant là une condition de l'expression de son intimité. Nous sommes plus habitués, à cause du principe discriminatoire de la lecture, à saisir un paysage par l'acte de se représenter ce qui le configure pour notre regard. Toute la didactique contemporaine de la lecture des paysages nous conduit à penser que rien ne nous échappe dans nos manières de percevoir n'importe quel territoire. Et le travail des architectes qui confectionnent des paysages va dans le même sens : la finalité est toujours la même, elle consiste à élaborer ce qui prédéterminera notre vision, même si l'incertitude de la configuration peut être entretenue grâce à une sorte de gestation propre du paysage ainsi conçu. Or, l'appréhension de l'irreprésentable ne peut être rendue possible que par l'émergence réelle ou virtuelle de la catastrophe.

Quand on cherche parmi les peintres des ruines ceux qui ont voulu représenter le mouvement même de la catastrophe, on ne trouvera peut-être que Monsu Desiderio. Je ne rappellerai pas l'histoire de ces deux peintres, François de Nomé et Didier Barra, qui ont parfois signé, leurs tableaux du seul nom de Monsu Desiderio, je considérerai surtout L'Explosion dans une église qui, de manière unique dans toute l'histoire de l'art, présente la mise en mouvement de l'effondrement. Sans aucun doute, la manière de saisir le temps même de la catastrophe est-elle une gageure dans la peinture. La photographie, le cinéma réussissent à fixer ce moment et à en offrir l'expectative au regard, mais la peinture ne peut le faire que de façon analogique dans le processus 
même de la représentation ou alors grâce à l'abstraction. L'Explosion dans une église est l'expression même de cette mise en suspension du mouvement de l'éboulement, quand celui-ci advient et qu'il peut se poursuivre.

Cherchant à explorer les différentes manières dont l'art traite la catastrophe, nous avons rencontré Christophe Buisson, qui peint des toiles en utilisant des cendres pour créer un effet d'opacité de la matière qui prend l'aspect d'une surface contaminée. Parmi ses œuvres, il a fait une série de huit tableaux «SARKOPHOGOS» tels des amas de peinture, de cendres et de fibres végétales, en partant d'une photographie sans aucun aspect narratif, en répétant une variation de motifs qui se dissolvent dans la matière picturale.

Curieusement, ses peintures "SARKOPHOGOS " ont été réalisées avant sa première visite à Tchernobyl avec nous. À première vue, elles représentent des paysages sinistrés que la matière rugueuse, épaisse, rend plutôt inquiétants par les teintes sombres, ocre rouge, noire, jaune. Il est frappé, nous a-t-il dit, par l'allure médiévale du sarcophage. Il se concentre sur une « idée picturale » et ne cherche pas l'élaboration d'un récit. Il se pose la question de l'irreprésentable dans la production de l'image qui méduse sans se soucier d'adopter une position didactique. Comment la disparition de la représentation du sarcophage de Tchernobyl se fait-elle dans l'effet de contamination du processus même de «se représenter "? Il ne s'agit pas de choisir l'abstraction pour elle-même, mais de mettre en œuvre cet effet de dissolution de la représentation comme un modalité mentale contemporaine de la perception des choses, du monde, de l'événement comme de la répétition elle-même. Il ne s'agit pas non plus, de considérer l'irreprésentable comme une catégorie qui viendrait s'inscrire au cœur de la vision telle la désignation d'une insupportable horreur - mais de mettre en œuvre la trajectoire de l'irreprésentable comme l'effet d'une dissolution par la contamination, par «l'irradiance », de la représentation elle-même.

17 Ce que Christophe Bisson révèle à la différence d'un peintre comme Kokian qui, lui, expose dans un style "graffitis " les effets horribles du nucléaire à Tchernobyl, c'est l'intensité d'une présence par sa disparition, par son irradiation, c'est la souveraineté du retrait par le motif qu'on n'atteint jamais et qui ne se retranche pas du côté du sublime. Ces peintures qui, dans le moment de la perception, peuvent paraître statiques, introduisent un mouvement plus abstrait, celui de la déprise : ce qui se donne à voir, se retire. La série des tableaux crée un effet de rythme, il s'agit, pour lui, d'éviter d'épuiser une réalité rhétorique par la puissance de l'image. En ce sens, il se distingue, toujours selon lui, d'Anselm Kiefer dont le rapport à l'histoire prend de manière déterminée un aspect très narratif, très construit malgré l'effet d'abstraction et de contamination du sens que produit sa peinture.

18 Les territoires sinistrés laissent penser à des matériaux qui travaillent, qui continuent à travailler et les matières utilisées, en séchant, deviennent minérales, rugueuses. Cette croûte minérale vient jouer contre l'image. L'idée dominante, presque obsessionnelle, est que la matière réduit le pouvoir de l'image. La matière se fait elle-même irreprésentable. Avec la crémation, la décomposition, le pouvoir de la médiatisation par l'image serait occulté. Christophe Bisson nous a dit avoir rêvé, en revenant de Tchernobyl, d'une grande arche dorée avec des peintures byzantines, qui fut, pour lui, une manière de mettre fin au rêve d'enfermement. Cette sérénité des lieux, ce silence, cette lumière, qu'il a ressentis sur le site même de Tchernobyl ont confirmé la 
puissance souveraine de l'irradiation de la représentation de l'horreur d'une manière qu'il refuse de considérer comme " esthétique ".

Entre la dissolution de la représentation et l'émergence des effets de catastrophe, les regards portés sur les paysages sinistrés peuvent par eux-mêmes avoir un rôle de réparation. Il ne s'agit plus d'un enjeu philosophique de la dissémination (Jacques Derrida), ni d'une réduction à l'esthétisation du "catastrophique ", c'est un espace « entre-deux » qui s'ouvre et dans lequel la « disparition » devient " apparition », cette dernière étant prise dans la forme abstraite de son expression, ou dans l'abstraction de son expression mentale, justement provoquée par la disparition elle-même. Cette question nous rappelle un phénomène de perception bien connu: quand il manque certaines choses dans un champ de vision auquel nous avons été habitués, nous restituons, sans même nous en apercevoir, ce qui n'est pas là, mais qui devrait y être. Il y a des effets de congruence dans l'acte de percevoir qui produisent des associations, des complémentarités, en rendant présent ce qui n'est pas là. C'est le même phénomène qui se passe dans la lecture quand nous rétablissons la présence d'une lettre manquante alors que nous ne nous sommes même pas aperçus qu'elle était absente. Reste à savoir si dans le regard porté sur un paysage catastrophé nous opérons de telles congruences. Et dans quelle mesure l'art, en répondant à sa vocation de réparation, ne crée-t-il pas d'autres moyens et d'autres effets de congruence? Et pourquoi la congruence seraitelle un processus de pacification de la perception?

\section{RÉSUMÉS}

Sans doute est-il outrecuidant de s'interroger sur le fait qu'un territoire sinistré puisse «faire paysage ». Si je prends pour exemple le site de Tchernobyl, je peux, bien entendu, m'attacher à la perception du «sarcophage ». Ce réacteur enveloppé par une chape de béton se présente dans le champ de ma vision comme le symbole majeur de la catastrophe passée. Il cristallise à lui tout seul toute la perception des lieux en imposant au regard le souvenir impérissable du désastre, de son histoire inoubliable. Mais en parcourant ces mêmes lieux, je vois aussi que la nature est devenue luxuriante, que des traces des villages disparus sont enfouies dans les hautes herbes, je sens que le site dans son ensemble est plongé dans un étrange silence... Toutes ces sensations adviennent simultanément comme si j'étais happé par une atmosphère dont chaque détail ne fait que conforter mon impression de densité du paysage. Les territoires sinistrés nous invitent à voir ce qui n'est pas en ce qui est, en ce qui demeure visible. Il ne s'agit pas des traces repérables qui permettent une lecture toujours possible de ce qui s'est passé, ou une mise en représentation d'une catastrophe ancienne. Le temps produit une dissolution de la représentation en imposant au regard la puissance de l'irreprésentable. Et la catastrophe n'est-elle pas ce qui, par essence, est irreprésentable?

It probably is presumptuous to wonder about the way a disaster area can "form a landscape". Using the site of Chernobyl as an example: I can of course linger on the perception of the "sarcophagus", this reactor wrapped in a concrete shell which appears before my eyes, the major symbol of the past catastrophe. It alone crystallizes the whole perception of this place, imposing to our vision the imperishable memory of the catastrophe, of its unforgettable history. But, 
walking around these places, I see also how nature has become luxurious, how the remnant traces of long gone villages are buried in the tall grass; I perceive how the entire site seems smothered in a strange silence... Disaster areas invite us to see what is not through what is visible, through what remains there to see. I don't mean the traces that can be spotted and which always render possible a reading of what has taken place, or a mediated representation of the old catastrophe. Time causes the dissolution of the representation, by forcing our gaze to take in the power of that which is impossible to represent.

Es wird hier vorgeschlagen zu sehen was man nicht mehr sehen kann, was davon sichtbar bleibt. Es handelt sich nicht um die noch erkennbaren Spuren, die eine mögliche Lektüre erlauben von dem was geschehen ist oder eine Inszenierung einer früheren Katastrophe. Die Zeit löst die Vorstellung auf und zwingt dem Blick die Macht des Unvorstellbaren auf. Und ist die Katastrophe nicht an sich unvorstellbar?

\section{AUTEUR}

\section{HENRI-PIERRE JEUDY}

Henri-Pierre Jeudy, sociologue au CNRS, écrivain, est l'auteur de nombreux ouvrages, parmi les quels Le Désir de catastrophe (Aubier, 1990), Le Corps comme objet d'art (Armand Colin, 1997), Fictions théoriques (Léo Scheer, 2003), La Machine patrimoniale (Circé/poche, 2008), L'Absence de l'intimité (Circé, 2007), Nouveau discours amoureux (Léo Scheer, 2008). 